\title{
Merkur: herramienta de transcodificación parametrizada de contenidos web para móviles
}

\author{
Por José-Manrique López-de-la-Fuente
}

\begin{abstract}
Resumen: Según algunos estudios, en 2009 el número de dispositivos web móviles será seis veces superior a los de escritorio o portátiles. A partir de estos datos se despiertan los intereses comerciales de los proveedores de contenidos de maximizar el alcance de sus materiales. Para ello se hace necesario el uso de mecanismos estandarizados de comunicación y que los contenidos sean accesibles de la forma más amplia posible. La Fundación Ctic (Centro Tecnológico de la Información y la Comunicación), experta en técnicas de estandarización y accesibilidad web a partir de la aplicación de las directrices del W3C, presenta la herramienta Merkur, que permite que páginas

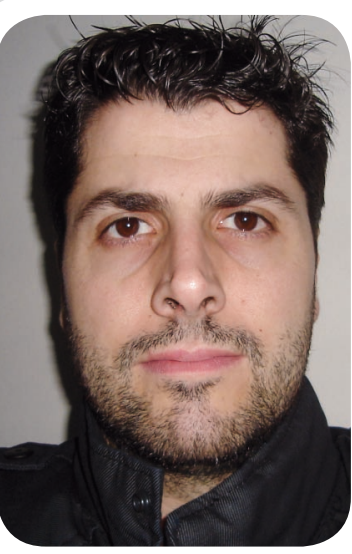

José Manrique López de la Fuente, ingeniero industrial (especialidad de gestión), es consultor, técnico e investigador en el Ctic (Centro Tecnológico de la Información y la Comunicación del Principado de Asturias). Es experto en movilidad web, participa en la W3C mobile web initiative y tiene experiencia en formación y difusión de estándares del W3DC (Web 3D Consortium). Participa en proyectos e iniciativas de ICT4D (TIC para el desarrollo) y M4D (movilidad para el desarrollo). Es miembro activo de las comunidades OpenSource, especialmente las centradas en dispositivos y soluciones móviles. Promotor de OpenSource y los estándares abiertos, así como de sus beneficios en innovación, educación y desarrollo.

construidas aplicando criterios de accesibilidad y estandarización web se adapten a dispositivos móviles siguiendo las pautas y recomendaciones de W3C de forma sencilla y eficaz.

Palabras clave: Movilidad web, Adaptación de contenidos, Transcodificación, Transformación de código

\section{Title: Merkur: custom transcodification tool for content adaptation to mobile devices}

Abstract: According to some studies, in 2009 the number of mobile web devices worldwide will be six times greater than that of desktop or laptop devices. These data have awakened the business interests of content providers to maximize the ways of access to their materials. For them, it becomes necessary to use standardized mechanisms for communication and to make content available as widely as possible. The Ctic Foundation (Center for Information Technology and Communication), experts in technical standardization and Web Accessibility based on implementation of the W3C guidelines, presents Merkur tool, which allows pages built following accessibility and standardization recommendations to adapt to mobile devices following the W3C guidelines and recommendations, in a simple and effective manner.
\end{abstract}

Keywords: Mobile web, Content adaptation, Transcodification, Code transformation

López-de-la-Fuente, José-Manrique. "Merkur: herramienta de transcodificación parametrizada de contenidos". El profesional de la información, 2009, marzo-abril, v. 18, n. 2, pp. 218-222.

DOI: 10.3145/epi.2009.mar.12

\section{Independencia web de dispositivo}

\section{LA WEB HACE POSIBLE} LA COMUNICACIÓN, el comercio y compartir conocimiento entre las personas.

Es deseable que estos beneficios estén disponibles para todos, sea cual sea el hardware, software, infraestructura de red, idioma nativo, cultura, localización geográfica o capacidades físicas o mentales de las personas. Desde el punto de vista técnico, "independencia web de dispositivo" significa poder acceder a la información de forma satisfac- toria sea cual sea el equipo utilizado. De esta forma se tendrían sitios web únicos, capaces, en la medida de lo razonable, suministrar la misma información y servicios a cualquier tipo de terminal receptor.

Esto no significa que se suministre la misma información con la misma representación a todos los equipos. Así, en el caso de los móviles se visualizará diferente según las capacidades de los mismos, las características de la red o el ancho de banda, y puede que algunos servicios sean más adecuados para ciertos contextos que otros.

\section{Iniciativa para la Web móvil del W3C}

Desde su creación, el $W 3 C$ ha trabajado en la definición de una serie de recomendaciones o estándares de carácter abierto que permitan garantizar el acceso a internet de forma efectiva por parte de los individuos. Siguiendo esta premisa se encuentra la Web accessibility initiative (WAI) en el marco de la cual se han redactado recomendaciones para el desarrollo de sitios web accesibles para cualquier persona independientemente de sus capacidades. 
Siguiendo con la misma filosofía, se ha creado la Iniciativa para la Web móvil de W3C (Mobile Web initiative o $M W I$ ), cuyo objetivo es también elaborar recomendaciones y estándares para que los sitios web garanticen a cualquier usuario una experiencia satisfactoria.

Para verificar su cumplimiento se ha confeccionado una serie de tests cuya finalidad es comprobar el nivel de adecuación de un sitio web a dispositivos móviles, definiendo un sello de garantía denominado mobileOK.

\section{Alternativas de webs optimizadas para móvil}

Se pueden aplicar diversas estrategias para conseguir sitios web que se visualicen correctamente en dispositivos móviles.

Lo más sencillo es construirlo desde cero, y seleccionar un gestor que soporte la producción de contenidos multicanal/multidispositivo y permita garantizar una experiencia de usuario adecuada para todos. Entre los problemas que presenta esta estrategia se encuentran:

- La curva de aprendizaje suele ser alta.

- Cuando el sitio web ya funciona hay que adaptar la nueva plataforma a la gestión de contenidos existente. Habitualmente los programas están optimizados para que sus resultados sean visualizados por plataformas concretas, con lo que la aparición de nuevos dispositivos con acceso a internet obliga a replantearse su utilización.

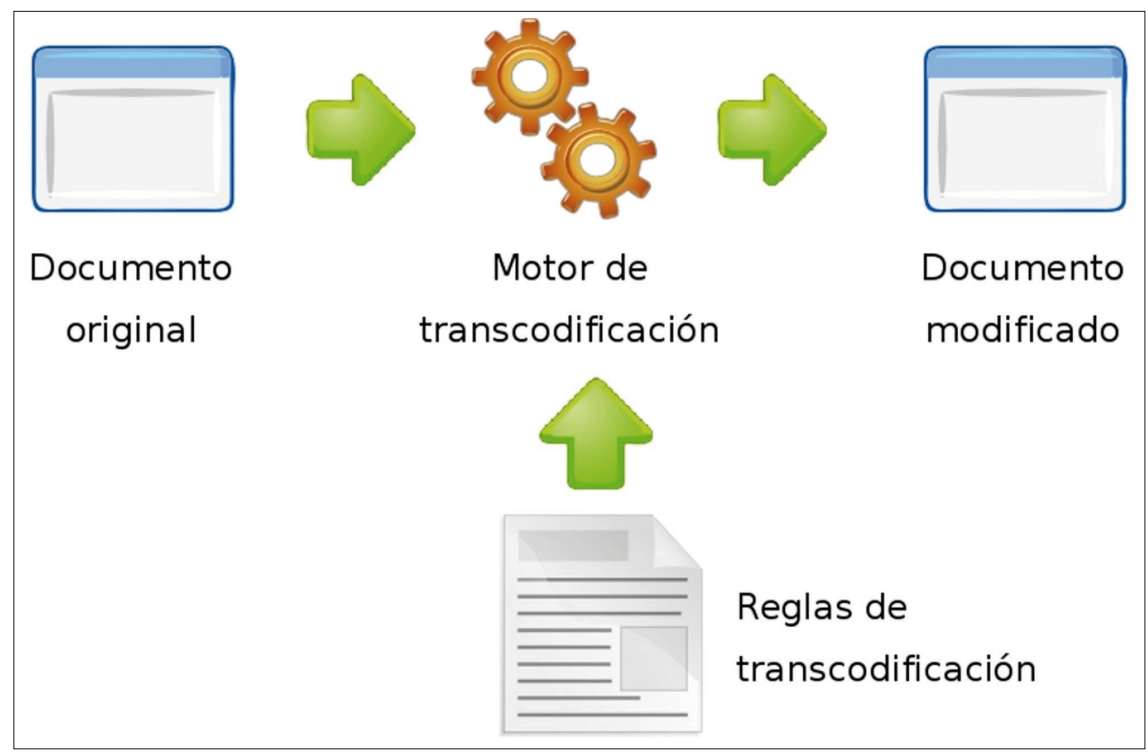

Figura 1. Proceso de transcodificación

El caso más frecuente es, precisamente, que el sitio web ya exista, y se desee ofrecer una correcta visualización del mismo en aparatos móviles con un impacto mínimo sobre la infraestructura ya existente. Una posible solución es dejar que aplicaciones de terceros adapten la página, lo que se denomina "transcodificación general". El proceso de transcodificación (figura 1) viene determinado por unas reglas fijadas en la aplicación, e imposibilitan que el responsable del sitio web controle el aspecto final del mismo en los diferentes dispositivos.

En resumen, se presentan varias estrategias cuyas ventajas e inconvenientes mostramos en la tabla 1.

A la vista de las estrategias presentadas la solución óptima pasaría por integrar el proceso de transcodificación en la gestión del sitio web existente, que permitiese al desarrollador del mismo controlar la presentación de la información, así como asegurar una buena funcionalidad a través del móvil. A esta solución la denominaremos "transcodificación parametrizada", y es la base de la herramienta Merkur.

\section{Funcionamiento}

El objetivo de la movilización de sitios web es permitir que el contenido de la web sea visualizado de forma correcta en cualquier equipo móvil.

Merkur es una aplicación de movilización de contenidos web basada en estándares internacionales $W 3 C$ que facilita el acceso a la Web mediante un dispositivo móvil y permite que el sitio web cumpla los requisitos necesarios para alcanzar la categoría mobileOK.

Merkur actúa como un proxy inverso (transparente para el usuario), que se interpone entre la petición y los contenidos generados por el servidor. Su funcionamiento básico (figura 2) es el siguiente:

\begin{tabular}{|l|l|l|l|}
\hline & \multicolumn{1}{|c|}{ Descripción } & \multicolumn{1}{c|}{ Ventajas } & \multicolumn{1}{c|}{ Inconvenientes } \\
\hline Web móvil específica & $\begin{array}{l}\text { Se crea una web paralela a } \\
\text { la existente optimizada para } \\
\text { dispositivos móviles }\end{array}$ & $\begin{array}{l}\text { Experiencia de usuario } \\
\text { satisfactoria a través del } \\
\text { móvil }\end{array}$ & $\begin{array}{l}\text { Complejidad de gestión. } \\
\text { Dificultad de adaptación a } \\
\text { nuevos dispositivos }\end{array}$ \\
\hline Transcodificación general & $\begin{array}{l}\text { La web es adaptada por } \\
\text { servicios intermedios externos } \\
\text { al gestor de la misma, o incluso } \\
\text { se relega la labor de adaptación } \\
\text { al dispositivo }\end{array}$ & $\begin{array}{l}\text { Fácil integración con el } \\
\text { gestor de contenidos } \\
\text { existente al ser } \\
\text { independiente del mismo }\end{array}$ & $\begin{array}{l}\text { Falta de control sobre la } \\
\text { presentación final del sitio web. } \\
\text { No se garantiza una } \\
\text { experiencia de usuario óptima }\end{array}$ \\
\hline
\end{tabular}




\section{"Merkur mejora la experiencia de usuario al acceder a la Web mediante un dispositivo móvil"}

- Cuando la petición desde el dispositivo llega a Merkur (1), éste identifica sus capacidades y pasa la petición inalterada al servidor (2), garantizando la transparencia del sistema.

- Cuando llega la respuesta del servidor, Merkur (3) en función de las capacidades del dispositivo y de la parametrización realizada, adapta los contenidos para optimizar la respuesta al móvil (4).

Merkur presenta ventajas sobre otras alternativas, como pueden ser:

- No es intrusivo ya que no afecta a la infraestructura existente en el sitio web donde se despliega por dos motivos: en primer lugar, al poder funcionar como un proxy puede instalarse en una máquina externa sin añadir carga de trabajo a la infraestructura existente. En segundo lugar, puede ser instalado sobre cualquier sistema operativo que soporte la plataforma Java.

- Es independiente de la plataforma de gestión de contenidos del proveedor.
- Hace posible que una única url ofrezca la misma información adaptada a diferentes dispositivos. Merkur está pensado para facilitar el concepto de "web única".

Sus componentes esenciales son:

- Un sistema de descubrimiento de capacidades basado en tecnologías estándar W3C y OpenSource que permite reconocer el equipo que hace la consulta.

- Un componente que filtra las páginas sobre las que se va actuar, evitando tratar aquellas que el gestor del sitio considere no adecuadas para dispositivos móviles.

- Dos sistemas independientes de transcodificación, uno para el lenguaje de marcado que define el sitio web, y otro para los elementos multimedia.

El sistema de transcodificación de marcado es parametrizable por el usuario mediante un sencillo lenguaje específico de dominio (domain specific language o $d s l$ ) desarrollado para la herramienta. El uso de un lenguaje de alto nivel como es un $d s l$, disminuye la curva de aprendizaje, aumentando la productividad. Este lenguaje hace posible el manejo de objetos comunes en la web (bloques, elementos, etc.) y realizar acciones sobre ellos como:

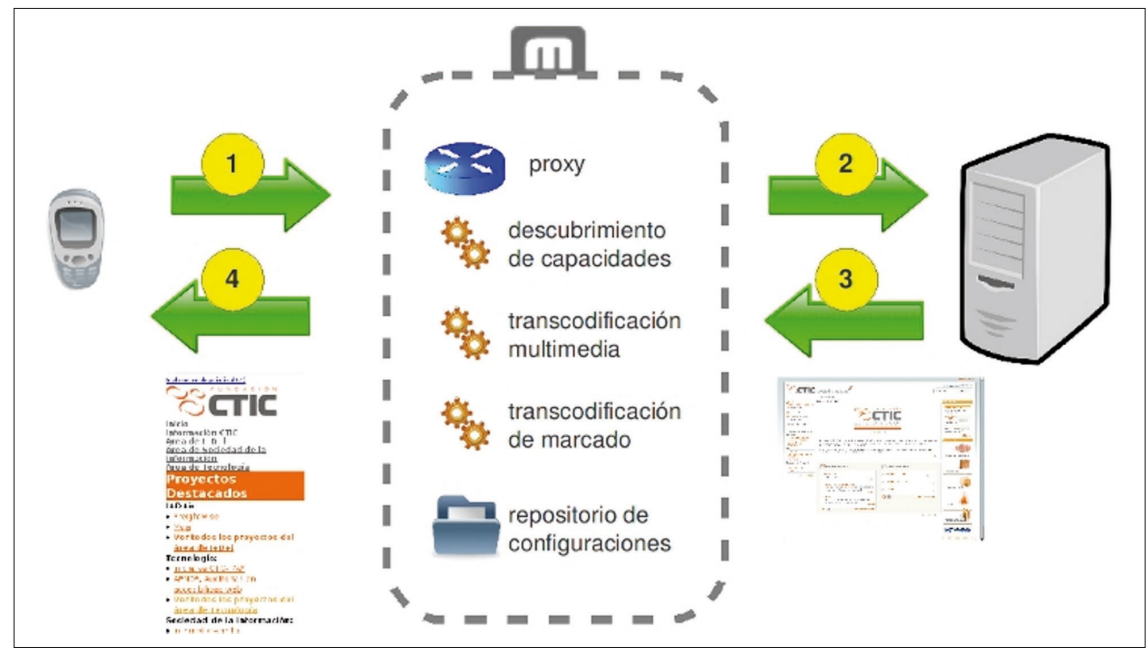

Figura 2. Esquema de funcionamiento de Merkur
- Eliminar elementos superfluos para el usuario de dispositivo móvil.

- Añadir nuevos elementos, sustituir imágenes por sus textos alternativos e incluso insertar enlaces telefónicos (que activan una llamada desde el móvil).

- Reubicar elementos dentro la página.

- Linealizar tablas según distintas estrategias.

- Personalizar las hojas de estilo (css) para dispositivos móviles y eliminar las no adecuadas.

- Traducir el lenguaje de marcado a uno preparado para móviles (xhtml basic o xhtml mobile profile).

- Partir páginas de forma inteligente para reducir la cantidad de datos enviados al dispositivo.

- Etc.

La adaptación de elementos multimedia se hace a través de un sistema de transcodificación específico. Por ejemplo, se adaptan las imágenes según los siguientes parámetros:

- en tamaño, para que no sobrepasen el ancho de pantalla del receptor reduciendo la cantidad de datos enviados y, con ello, los tiempos de descarga necesarios.

- en formato, convirtiendo las imágenes de formato no soportado por el dispositivo al indicado como formato preferido.

Cada parametrización hecha para un portal se almacena en un repositorio de configuraciones que Merkur utiliza. Se convierte así en una herramienta multiportal, de manera que una organización o empresa que gestione varios sitios web puede aplicarla de forma independiente sobre cada uno de ellos.

\section{Herramienta visual de configuración de Merkur}

Para facilitar aún más el proceso de configuración de Merkur, 


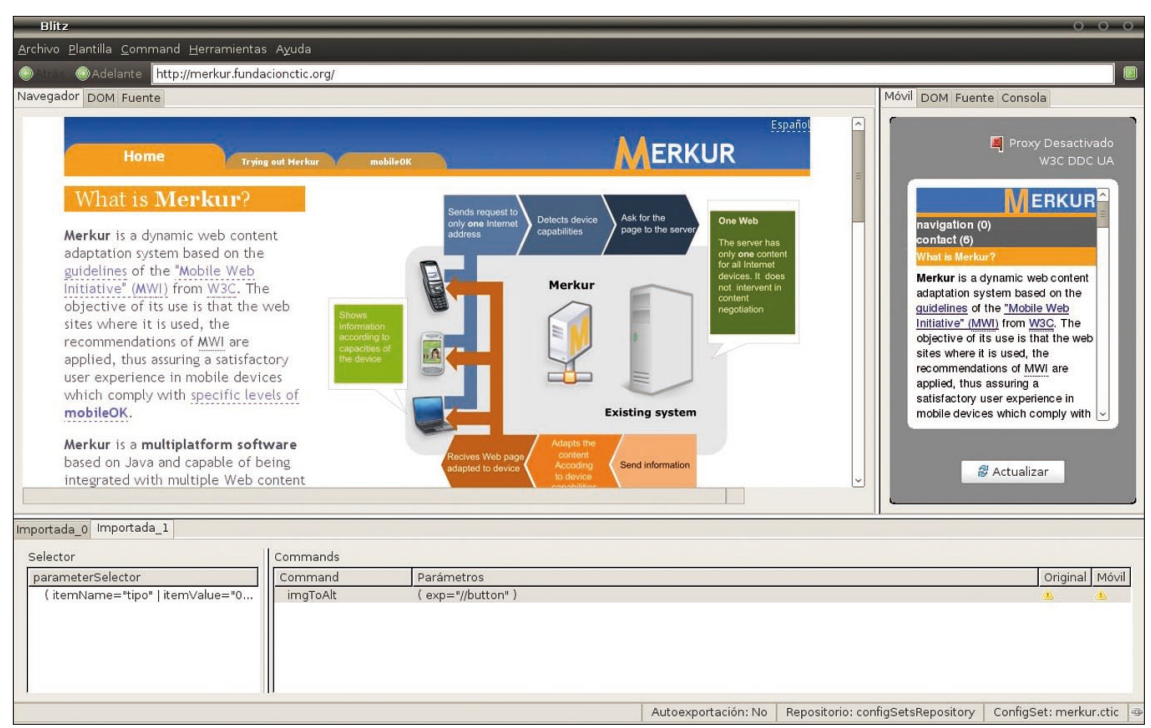

Figura 3. Blitz

se ha desarrollado una herramienta visual que actúa como asistente de ayuda para crear la parametrización necesaria para cada sitio web que se quiera movilizar (figura 3).

Esta aplicación, denominada Blitz, permite actuar de forma visual sobre los elementos de una web existente, y obtener una previsualización en tiempo real de cómo se vería en un dispositivo móvil.

\section{Transferencia tecnológica}

La Fundación Ctic ha acumulado un know-how importante en tecnologías de estandarización web, fruto de su estrecha colaboración con los grupos de investigación del $W 3 C$, consorcio mundial en el que se integra. Ese conocimiento se pone al servicio de instituciones y empresas para implementar arquitecturas web basadas en estándares internacionales, con el objeto de conseguir una Web universal, interoperable, móvil, accesible e inteligente.

Por las características organizacionales de la Fundación Ctic, el modelo de negocio para una solución como Merkur consiste en una transferencia tecnológica de la herramienta al cliente bajo el siguiente formato:

- Formación del cliente en el conocimiento de las pautas y reco- mendaciones del $W 3 C$ para estandarización web, y especialmente en el campo de la "independencia de dispositivo".

- Implementación de un caso práctico de adaptación de contenidos, para que el cliente conozca la aplicación y cómo seguir con ella las recomendaciones del $W 3 C$.

- Revisión de las realizaciones de los clientes de Merkur de cara a asegurar el cumplimiento de todas las pautas y recomendaciones del W3C en el ámbito de la "independencia de dispositivo".

Con esta transferencia, el cliente adquiere conocimiento sobre "independencia de dispositivo" acorde con los estándares promovidos desde el W3C y una aplicación completa para aprovecharlo.

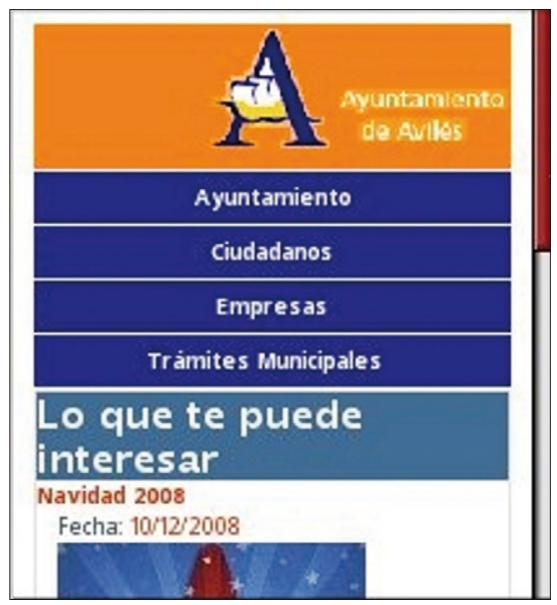

Figura 4. Ayuntamiento de Avilés
Esta modalidad ya se está utilizando en diversos sitios web como Ayuntamiento de Avilés (figura 4), Ayuntamiento de Zaragoza (figura 5), Ticmanía (figura 6) y Ayuntamiento de Gijón (figura 7).

Además, tanto el sitio web de la Fundación Ctic como el del proyecto Merkur están adaptados a dispositivos móviles con la misma herramienta.

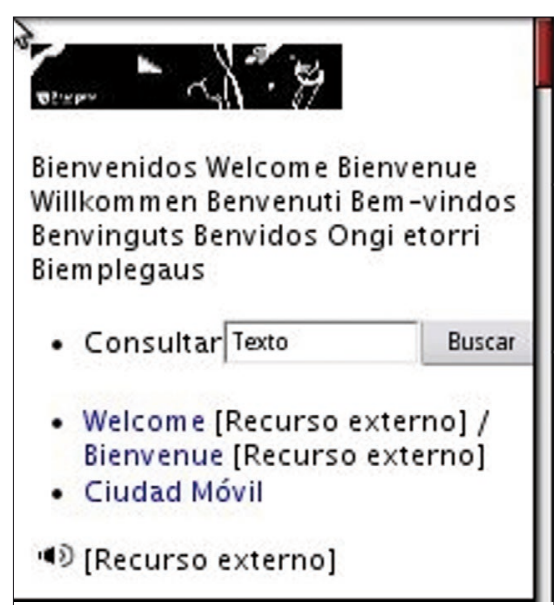

Figura 5. Ayuntamiento de Zaragoza

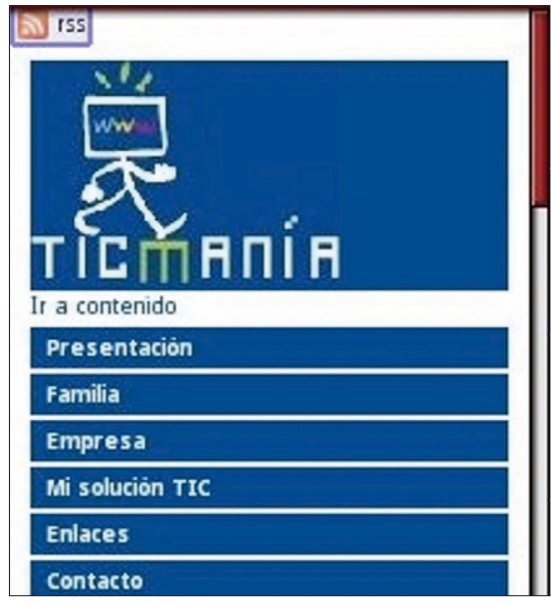

Figura 6. Ticmanía

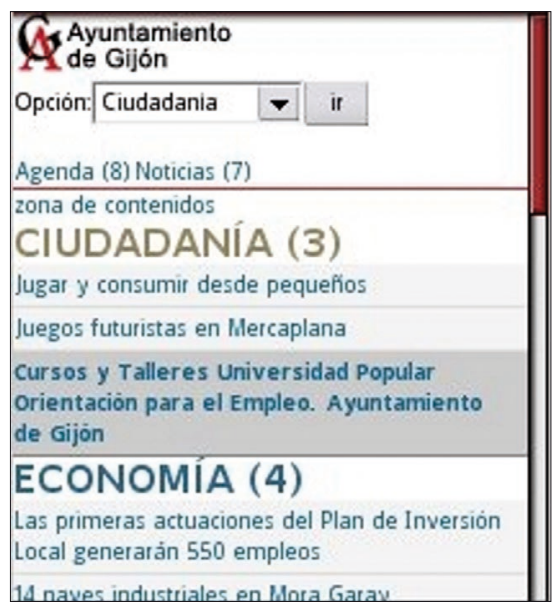

Figura 7. Ayuntamiento de Gijón 


\section{Acerca de la Fundación Ctic}

La Fundación Ctic es una institución privada, sin ánimo de lucro, de carácter social y de cooperación para el desarrollo tecnológico. Está constituida por un patronato de empresas del campo de las tecnologías de la información y la comunicación (TIC) y por el gobierno del
Principado de Asturias. Su objetivo fundacional es promover y estimular el desarrollo de las TIC en todos los campos de la vida económica y social, que conduzcan al fortalecimiento de la sociedad de la información.

http://www.fundacionctic.org http://merkur.fundacionctic.org
José-Manrique López-de-la-Fuente, Área de Tecnología, Fundación CTIC, Parque Científico Tecnológico de Gijón, Edificio Centros Tecnológicos, Cabueñes s/ $n$.

33203 Gijón, Asturias, España.

Tel.: +34-984291212

manrique.lopez@fundacionctic.org http://www.fundacionctic.org

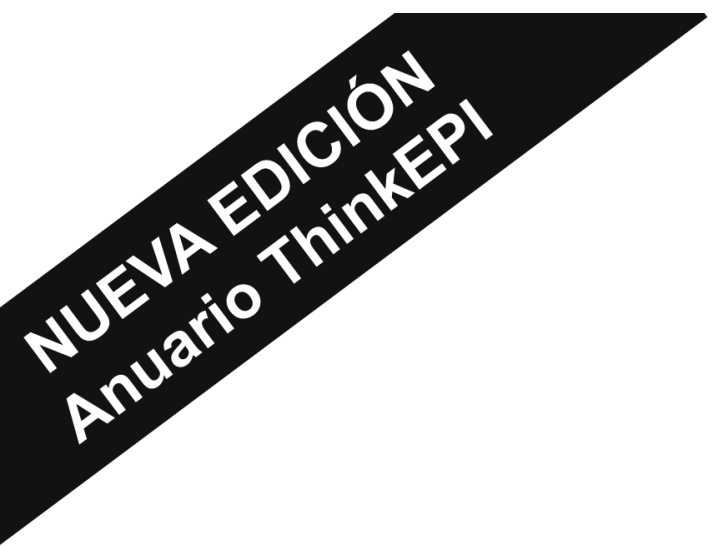

\section{9 Análisis de tendencias en información y documentación}

\section{Os avanzamos algunos de los títulos:}

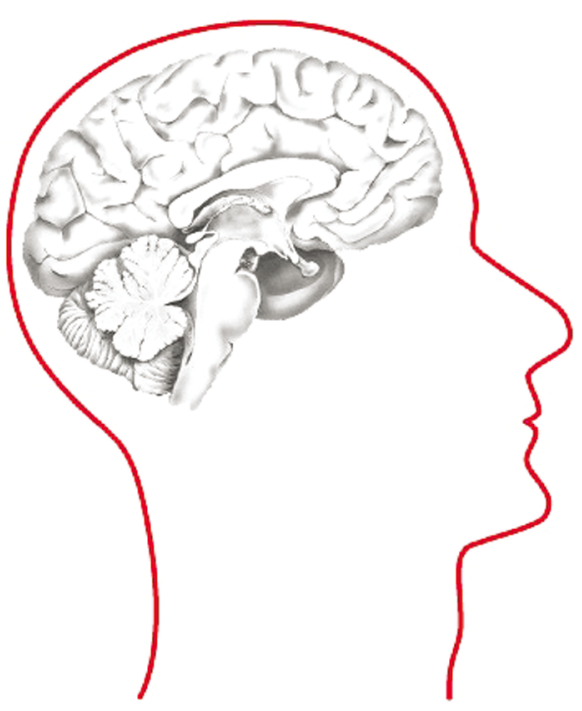

El uso profesional de las redes sociales Natalia Arroyo

Movilidad de la información: de la biblioteca al salón de casa

Pablo Lara-Navarra

Las universidades y el apoyo institucional al Open access

Ernest Abadal y Reme Melero

Biblioteca 2.0: ¿revolución o nuevo maquillaje para viejas formas de hacer?

Roser Lozano

El investigador y el editor ante la evaluación de revistas científicas

Elea Giménez-Toledo

\section{ANUARIO}

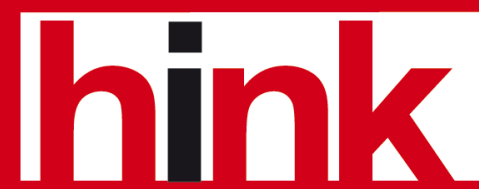

